\title{
The immunomodulatory activities of licorice polysaccharides (Glycyrrhiza uralensis Fisch.) in CT 26 tumor-bearing mice
}

Peter Amwoga Ayeka ${ }^{1,2}$, YuHong Bian ${ }^{1 *}$, Peter Mwitari Githaiga ${ }^{1,3}$ and Ying Zhao ${ }^{1}$

\begin{abstract}
Background: The increasing use of complementary and alternative medicine (CAM) has kindled the need for scientific evaluation of the mechanism of action of CAMs. Although, licorice, a common ingredient in many Traditional Chinese medicine (TCM) has attracted great attention for its antitumor and immunomodulatory activities, the mechanism of action of its polysaccharides is still unclear. Here we report the immunomodulatory activity of licorice polysaccharides in vivo.
\end{abstract}

Methods: The differential anticancer activities of licorice polysaccharides by tumorigenesis and immunomodulation was evaluated in vivo. Six weeks old, 120 CT-26 tumor bearing BALB/c mice, weighing $20 \pm 2 \mathrm{~g}$ were used. They were randomly divided into six groups, three groups receiving high molecular weight (fraction A), low molecular weight (fraction B) polysaccharides and crude extract (fraction C); positive, negative and normal groups receiving cytoxin, saline and normal diet respectively. Weight of mice and tumors was determined and tumorigenicity assay calculated to determine the anticancer effects. Immunomodulatory potential was determined by immune organ indices, immune cell population and serum cytokine levels using immune organ weight and index, flow cytometry and cytokine/chemokine bead panel kit respectively.

Results: Licorice polysaccharides exhibited immunomodulatory activities in CT 26 tumor bearing BALB/C mice. The polysaccharides significantly suppressed tumor growth and increased immune organ index. Furthermore, the immunomodulatory effect was evident with activation of $\mathrm{CD}^{+}$and $\mathrm{CD} 8^{+}$immune cells population. The polysaccharides also affected the production of various cytokines, by increasing IL 2, IL 6, IL 7 levels and a decreasing TNFa levels.

Conclusion: In summary, licorice polysaccharide especially of low molecular weight exhibit anticancer and immunomodulatory activities by suppressing tumor growth and improving general health of mice. They also augment the thymus/spleen index and population of T lymphocytes. Furthermore, the polysaccharides enhance the levels of serum antitumor cytokines, IL 2, IL 6 and IL 7 while decreasing pro-tumor cytokine TNFa.

Keywords: Licorice, Immunomodulation, Anticancer, Polysaccharides, Tumor

\footnotetext{
* Correspondence: bianyuhong_2012@163.com

${ }^{1}$ International College of Chinese Materia Medica, Tianjin University of

Traditional Chinese Medicine, 88 Yuquan Road, 312 Anshan Western Road,

Nankai District, Tianjin 300193, People's Republic of China

Full list of author information is available at the end of the article
} 


\section{Background}

According to global cancer burden, cancer is one of the leading causes of death worldwide, and in 2013, it was rated the second after cardiovascular diseases. With an estimated $70 \%$ increase in new cases and death, it is estimated that it will rise from 12.7 million to 21.4 million new cases and 13 million cancer death by the year 2030 [1-3]. Due to the burden caused by cancer and side effects associated with target therapies, researchers have focused on better strategies to treat and manage cancer. Currently, researchers have focused on cancer immunotherapy, which pose mild and manageable effects than side effects associated with conventional therapies [4, 5]. The immune therapies are either applied individually or combined with or after chemotherapy, radiotherapy and surgery $[4,6]$. Immunomodulation in cancer therapy involves immune cells that are actively involved as antigen presenting cells (APCs), activation of T lymphocytes, cytokine production, suppression of inflammation and direct cytotoxicity to cancer cells $[5,7]$. Therefore, compounds with immunomodulatory activity are of great importance in cancer immunotherapy.

Plant based extracts including those from fungi, algae and lichens have elicited great passion among researchers due to their immunomodulatory and anticancer potential. Of importance, is Licorice (Glycyrrhiza uralensis Fisch.), a promising immunomodulatory and anticancer herb and a common ingredient in major prescriptions in Chinese medicine. Its pharmacological important compounds include triterpenes, saponins, flavonoids and phenolic compounds among others [8, 9]. In Our previous studies, for instance, licorice polysaccharides exhibited anticancer and immunomodulatory potential in vitro. The polysaccharides up-regulate expression of antitumor cytokine, IL-7, enhance secretion of IL-7 by mouse intestinal epithelial cells (IEC) and stimulates $\mathrm{T}$ lymphocytes [10]. Isoliquiritigenin, glycyrrhizin and glabridin, licorice compounds, show antiproliferative and apoptotic properties by acting directly on cancer cells in vitro [11, 12]. Immunomodulation potential of licorice compounds is also exhibited by $18 \beta$-glycyrrhetinic acid which up-regulates $\mathrm{T}$ cell proliferation, increase blood leukocyte count and spleen weight in mice [13-15]. These compounds have also been found to enhance apoptosis in cancer cells through upregulating c-myc and c-jun oncogenes and increasing activity of protein kinase C [16]. Licorice polyphenols, liquiritin, isoliquiritin and isoliquiritigenin, have demonstrated varying effects that range from cytotoxicity, apoptosis via caspase and $\mathrm{Bcl}-2$ cascades, augmenting expression of Bax and Bid proteins and downregulating Bcl-2, which are initiated through caspase pathway [17]. In $4 \mathrm{~T} 1$ tumor model, licorice extracts inhibit metastasis of cancer cells and delay tumor progression. They also reduce tumor growth, inhibit tumor angiogenesis, inflammation and lymphangiogenesis $[18,19]$, which is an essential attribute for a pharmacological cancer drug. In addition, concurrent use of licorice compounds and chemotherapeutic treatment of cisplastin show increased anticancer activity and hepatoprotective potential in mice and rats [20, 21]. Furthermore, licorice extracts have the ability to downregulate expression of proinflammatory cytokines, tumor necrotic factor alpha (TNF $\alpha$ ), interleukin 1 (IL-1) and interleukin 6 (IL-6) [22]. Despite this activities, immunomodulatory mechanism of action of licorice polysaccharides in vivo is still unclear. In this study, we demonstrate the immunomodulatory activities of licorice polysaccharides in CT26 tumor bearing mice.

\section{Methods}

\section{Materials and animals}

All chemical reagents were purchased from Sigma (Sigma-Aldrich Inc., St. Louis, MO, USA unless otherwise stated). Licorice (Glycyrrhiza uralensis) extracts were extracted and provided by the TUTCM herbal pharmaceutical company, Tianjin, China. The polysaccharides were fraction A of over $100 \mathrm{kDa}$ was $81.4 \%$ (High molecular weight), fraction $\mathrm{B}$, of $75 \mathrm{kDa}$ was $45.4 \%$ and $54.6 \%$ polysaccharide fractions was under $10 \mathrm{kDa}$ (Low molecular weight) and fraction C, $34.5 \%$ polysaccharide fractions was $290 \mathrm{kDa}$ and $14 \mathrm{kDa}$ was $30.3 \%$, total crude extract. BALB/c CT-26 tumor bearing mice (Institute of animal research in Beijing, China).

\section{Preparation of licorice polysaccharides}

Briefly, dried roots of Licorice (Glycyrrhiza uralensis) (1000 g) was extracted by boiling with $95 \%$ ethanol $(v / v)$ for $2 \mathrm{~h}(\mathrm{~h})$. The mixture was filtered and air dried and extracted with $10 \% \mathrm{v} / \mathrm{v}$ boiling water twice, $2 \mathrm{~h}$ each time followed by centrifugation. The aqueous extract was concentrated to $1.5 \mathrm{~L}$ under vacuum. Subsequently, 95\% ethanol was added to the aqueous extract up to $80 \%$ to precipitate the polysaccharides and kept at $4{ }^{\circ} \mathrm{C}$ overnight. After centrifugation, the crude licorice polysaccharides (LP) were dried by forced air at $50{ }^{\circ} \mathrm{C}$ and weighed. The crude LPs were completely dissolved in aqueous solution at $5 \%$ followed by ultraphonic for $1 \mathrm{~h}$ $\left(80 \mathrm{HZ}, 30^{\circ} \mathrm{C}\right)$ and stirred for $4 \mathrm{~h}$ at room temperature. The crude LPs solution was centrifuged at $6000 \mathrm{rpm} /$ min for $10 \mathrm{~min}$. The supernatant was enriched and divided into 3 fractions (GP-A, GP-B and GP-C) for alcohol precipitation by addition of $95 \%$ ethanol with stirring homogeneously for $2 \mathrm{~h}$. The final alcohol concentration was different for the 3 fractions (LP-A: 57\%; LP-B: 57 82\%; LP-C: $82 \%$ ) and kept at $4{ }^{\circ} \mathrm{C}$ overnight. Polysaccharide pellets were obtained by suction filtration and repeatedly washed sequentially with 
anhydrous ethanol, acetone and diethyl ether respectively. The proteins in the extract were removed using the Savage reagent. The LPs solutions of 3 fractions were dialyzed against distilled water for $36 \mathrm{~h}$ and freeze dried for further experiments.

\section{Antitumor activity of G.uralensis polysaccharides in vivo} Use of animals in this study was approved by Tianjin University of Traditional Chinese Medicine Animal Care and Use Committee. One hundred and twenty (120) CT26 tumor bearing BALB/c mice, 6 weeks old weighing on average $20 \pm 2 \mathrm{~g}$ were used. The animals were housed in an animal facility well light by natural light, well ventilated and temperature maintained at $22 \pm 2{ }^{\circ} \mathrm{C}$. They were kept in groups of ten mice per cage and received normal mice feed and water ad libitum. Animal care and handling was performed in accordance with the guidelines for animal experiments approved by the animal care and use authorization committee of TUTCM. After three (3) days of acclimatization, mice were randomly sorted into six groups of twenty mice for further experimentation. Three fractions of licorice extracts (Fraction A, B and C) were orally administered to mice, $500 \mathrm{mg} /$ $\mathrm{kg}$ once daily for 14 days. Cyclophosphamide (Cytoxin) was administered intraperiteonal at $50 \mathrm{mg} / \mathrm{kg}$ of mice for 10 days as positive control, the vehicle was given to negative control and normal mice received normal diet.

\section{Effect of polysaccharides on health of mice}

The weight of the animals were taken at $0,5,10$ and 15 th day. After the treatment period, mice were sacrificed and tumor weight in each group recorded. The net weight of mice was determined by subtracting tumor weight from final body weight of mice. The changes in body weight of mice in different groups were also evaluated. General health of mice was closely monitored by observing activeness, grooming and feeding behaviour.

\section{Euthanasia of mice}

After taking weight and observing general health, mice were euthanised and sacrificed for successive assay. Briefly, intraperitoneal injection (IP) of sodium pentobarbital $(250 \mathrm{mg} / \mathrm{kg})$ was administered to the mice [23]. This was followed by excision of tumors and immune organs.

\section{Tumorigenicity assay}

Tumor growth was monitored for 14 days after which the animals were sacrificed. The tumor suppression ratio was determined by the following formula:

Tumor suppression $(\%)=[(\mathrm{A}-\mathrm{B}) / \mathrm{A}] \times 100$. Where $\mathrm{A}$, is average tumor weight of the negative control, $\mathrm{B}$, is the weight of the treatment group.

\section{Relative immune organs weight}

After treatment period, mice were sacrificed, and the spleen and thymus weight and indices were measured and calculated. The thymus and spleen indices were expressed as thymus or spleen weight relative to body weight.

\section{Immune cells population}

One hundred and twenty CT-26 tumor BALB/c mice were equally randomised into six groups. Each group was treated with the corresponding treatment according to the experimental design for 14 consecutive days. To determine the effect of the polysaccharides on immune cell population, blood was drawn by retro-orbital puncture after treatment period. T lymphocytes subsets (CD4 ${ }^{+}$and $\mathrm{CD}^{+}$) from peripheral blood were measured and analysed on FACS Calibur. Briefly, whole blood was drawn into EDTA pre-coated $1.5 \mathrm{ml}$ tubes by rectalorbital puncture and mixed, $100 \mu \mathrm{l}$ added to the bottom of a well labelled tube and appropriate primary antibody added to each tube; to $100 \mu \mathrm{l}$ sample was added $0.25 \mu \mathrm{g}$ of FITC Hamster Anti-Mouse CD3e, $0.25 \mu \mathrm{g}$ of APC Rat Anti-Mouse CD4 and $1.0 \mu \mathrm{g}$ of PE Rat Anti-MouseCD8a (Biolegend Co. Ltd). The tubes were capped, vortexed and incubated in the dark for $25 \mathrm{~min}$ at room temperature, followed by addition of $1.5 \mathrm{ml}$ lysing solution vortexed, incubated at room temperature in the dark for 10-15 min and centrifuged for $5 \mathrm{~min}$ at $200 \mathrm{x}$ g. The supernatant was aspirated; cells fixed by re-suspending in $2 \%$ paraformaldehyde buffer for $30 \mathrm{~min}$ at room temperature and washed. The cells were finally suspended in $500 \mu \mathrm{l}$ wash buffer and stored for $24 \mathrm{~h}$ at $4{ }^{\circ} \mathrm{C}$ in the dark. A negative control of $50 \mu \mathrm{l}$ of cells plus $100 \mu \mathrm{l}$ PBS following the same procedure, was used. The samples were then analysed on FACS Calibur (Merck-Millipore, Bio-Rad Beijing) with InCyte software version 2.2.2.

\section{Serum cytokine levels}

To determine the effect of licorice polysaccharides on cytokine production, whole blood was drawn from mice after last day of treatment by rectal-orbital puncture, centrifuged at $4{ }^{\circ} \mathrm{C}$ for $3000 \times \mathrm{g}$ for $15 \mathrm{~min}$, serum obtained and frozen at $-20{ }^{\circ} \mathrm{C}$ until use. The cytokine levels in serum were measured by mouse cytokine/chemokine magnetic bead panel kit 96-Well Plate Assay (Milliplex MAP) Shanghai, China. The assay was run on Luminex

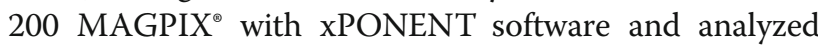
for Median Fluorescent Intensity (MFI) data using a 5-parameter logistic or spline curve-fitting method for calculating cytokine/chemokines concentrations in samples.

\section{Statistical analysis}

Data were expressed as Mean \pm SD. Normality of data was checked by Shapiro-wilk test and data was normally distributed. Statistical comparison between groups was 
carried out using one-way ANOVA followed by separation of means by Bonferroni. This was performed using SPSS Version 20.0. Curve-fitting was done using GraphPad Prism version 5 package. Values were considered statistically significant at $P$-value $<0.05$.

\section{Results}

\section{Mice weight and health}

After emergence of tumors and commencement of treatment, there was remarkable change in the general health of the treated mice. This was observed through state of grooming, activity and feeding behaviour. As shown in Table 1, tumor mice treated with licorice polysaccharides, fraction $\mathrm{B}$, had an increase in weight, $25.03 \pm 4.3 \mathrm{~g}$ and a low tumor weight of $5.96 \pm 0.8 \mathrm{~g}$. It had minimal change in weight and physical appearance. It's activity, grooming, eating and fur appearance did not change compared to saline group, which were emaciated and dull. The other treatment groups including the cytoxin group, had a reduction in total weight with saline group having highest weight loss. Notably, the results indicated that, although there was initial weight loss in all groups, there was a clear change in this trend, with the treated groups gaining weight. Fraction B treated group, weight $(19.07 \mathrm{~g})$, had the highest final weight compared to saline group, although the weight was lower than that of normal mice $(20.56 \mathrm{~g})$.

\section{In vivo tumor suppression}

To investigate in vivo antitumor activity of licorice polysaccharides, a successful model of CT-26 tumor bearing BALB/ c mice were treated orally with various concentrations for 14 days. The three licorice polysaccharide fractions showed varied tumor suppression rates. From the results in Table 1 above, Fraction B, had a significantly higher tumor suppression rate among all groups albeit, lower than cytoxin treated group, that is, $19.57 \%$ and $31.85 \%$ respectively while fractions A had the lowest tumor suppression rate.

\section{Relative immune organ weight and indices}

Immunomodulatory activity of licorice polysaccharides was evaluated by determining their effect on immune organs, thymus and spleen. There was a significant increase in spleen and thymus weight $(p \leq 0.05)$ in fraction $B$ treated group compared to saline group. There was also a significant change in spleen and thymus indices. Fraction B had a significant high thymus index of 0.637 \pm 0.14 compared to saline treated group. Although, there was no significant difference in spleen index between all the groups, the highest spleen index was observed in Fraction B treated group (Table 2).

\section{Immune cell population}

The immunomodulatory effect via activation of $\mathrm{T}$ lymphocytes by licorice polysaccharides in CT-26 BALB/c tumor model mice was analysed by flow cytometry. After treatment period, $\mathrm{T}$ lymphocytes were analysed from peripheral blood and the relative levels of $\mathrm{CD} 4^{+}$ and $\mathrm{CD}^{+} \mathrm{T}$ lymphocytes subsets determined. From the results (Table 3 and Fig. 1), there was a significant increase in the percentage number of $\mathrm{CD}^{+} \mathrm{T}$ lymphocytes subset in all the groups except Fraction C. Fraction B treated group showed the highest percentage of $78.70 \pm$ $3.4 \%$, saline group $75.74 \pm 4.7 \%$ and normal group $79.82 \pm .1 .1 \%$, with Fraction $\mathrm{C}$ having the lowest count of $71.20 \pm 0.9 \%$. The percentage population of $\mathrm{CD}^{+}$ subset of $\mathrm{T}$ lymphocytes was significantly reduced in all groups except Fraction $\mathrm{C}$ which showed a significant increase.

\section{Cytokine serum levels}

We set to evaluate antitumor potential of licorice polysaccharides through immunomodulation by measuring various peripheral cytokines (Fig. 2). After treatment period, cytokines, Interleukin 2, IL-6, IL-7, IL-10, IL-15, $1 \mathrm{~L}-17$ and TNF $\alpha$ in CT-26 tumor bearing BALB/c mice serum were analysed. The cytokine levels were measured by magnetic bead panel assay. From the results (Fig. 2), IL-2 levels increased in all treatment groups. Fraction B had the highest increase compared to other treatment groups except cytoxin treated group, even though the difference was not significant at $p<0.05$. The serum levels of IL-6 were low in all the treatment groups except for fraction B which had significantly higher

Table 1 Effect of licorice polysaccharides on weight of mice and tumor suppression

\begin{tabular}{|c|c|c|c|c|}
\hline Treatment Group & Mice with tumor(g) & Tumor weight(g) & $\begin{array}{l}\text { Mice without } \\
\text { tumor(g) }\end{array}$ & Tumor suppression (\%) \\
\hline Normal & $20.56 \pm 1.3$ & 0 & $20.56 \pm 1.3$ & - \\
\hline Saline & $23.48 \pm 2.7$ & $7.41 \pm 0.4$ & $16.07 \pm 2.3^{*}$ & - \\
\hline Cytoxin & $23.51 \pm 3.2$ & $5.05 \pm 1.1^{\#}$ & $18.46 \pm 2.1$ & 31.85 \\
\hline Fraction A & $25.60 \pm 2.4^{*}$ & $7.31 \pm 0.9$ & $18.32 \pm 1.5$ & 1.35 \\
\hline Fraction B & $25.03 \pm 4.3^{*}$ & $5.96 \pm 0.8^{\#}$ & $19.07 \pm 3.5$ & 19.57 \\
\hline Fraction C & $24.74 \pm 2.5$ & $6.59 \pm 1.0$ & $18.19 \pm 1.5$ & 11.07 \\
\hline
\end{tabular}

Data represented as mean $\pm S D, n=10 .{ }^{*} p<0.05$ versus Normal group; ${ }^{\#} p<0.05$, versus Saline group 
Table 2 Effect of Licorice polysaccharide on immune organs index

\begin{tabular}{lllllll}
\hline Index & Normal & Saline & Cytoxin & Fraction A & Fraction B & Fraction C \\
\hline Spleen mg & $80 \pm .002$ & $220 \pm .015$ & $160 \pm .028$ & $200 \pm .010$ & $250 \pm .029^{*}$ & $190 \pm .014$ \\
Thymusmg & $26.63 \pm 2.10$ & $9.87 \pm .66$ & $10.2 \pm .71$ & $13.56 \pm .54$ & $15.42 \pm .44^{*}$ & $13.67 \pm .53$ \\
Spleen index & $.004 \pm .00$ & $.009 \pm .00$ & $.007 \pm .00$ & $.008 \pm .00$ & $.010 \pm .00$ & $.008 \pm .00$ \\
Thymus index & $1.29 \pm .26$ & $.423 \pm .08$ & $.433 \pm .06$ & $.532 \pm .06$ & $.637 \pm .14^{*}$ & $.555 \pm .07$ \\
\hline
\end{tabular}

Data expressed as mean \pm SD.* $p<0.05$ versus saline group. $n=10$

levels of $28.31 \pm 1.2 \mathrm{pg} / \mathrm{ml}$ at $p<0.05$. In comparison to saline group, all treatment groups showed a significant increase except the cytoxin group. Furthermore, all treatment groups had an increase in the levels of IL-7 except Fraction A. That is, fraction B, the highest with $8.54 \pm 1.7 \mathrm{pg} / \mathrm{ml}$ and fraction A, lowest with $1.78 \pm$ $0.5 \mathrm{pg} / \mathrm{ml}$. The serum levels of the other cytokines including IL-10, IL-15 and 1 L-17 were lower in all the groups,except fraction A compared to saline group. When compared with the normal group, fraction B had higher levels of IL-15 and IL-17, with IL-10 being significantly high. The serum levels of TNF $\alpha$ were high in all the groups except fraction B and the normal group.

\section{Discussion}

Licorice plays an important role as an ingredient in many prescriptions used in complementary and alternative medicine. In TCM, it is prescribed for many diseases while in Japan, apart from its therapeutic use, it is also used as an ingredient in confectionary. Although the therapeutic potential of licorice have been studied, extensive work on immunomodulation and anticancer potential of its polysaccharides have not been fully exploited.

This study investigated the potential of licorice polysaccharides as immune modulating agents in CT 26 tumor bearing BALB/c tumor mice models. In our previous studies, we demonstrated that licorice polysaccharides not only upregulate IL-7 expression but also inhibit proliferation of cancer cells in vitro [10]. Augmenting of IL-7 expression is of great importance in cancer immunotherapy. This is due to the fact that IL-7 is a critical cytokine in lymphopoiesis. Besides IL-7, there have been concerted efforts to use the immune system to fight cancer, which has led to study, exploitation and development of other cytokines for cancer immunotherapy. Immunomodulation in cancer immunotherapy, as an emerging therapy is associated with minimal side effects, and boosts the overall body's immune system [24]. Therefore, agents that modulate the immune system are critical and important in the fight against cancers as opposed to conventional therapies that lead to immunosuppression.

Conventional therapies including radiotherapy, chemotherapy and surgery pose adverse secondary effects that ultimately lead to reduced quality of life, poor feeding habits, weight loss due to cachexia, recurrence of tumors and death, unlike immunotherapy that pose mild side effects and improve quality of life of patients [25-31]. In the recent past, there has been an increase in the use of complementary and alternative medicine due to its holistic treatment, less side effects and boosting of the immune system.

In this study, licorice, a common CAM, augmented the immune system in tumor mice models. The experimental mice treated with low molecular weight icorice polysaccharide, (Fraction B) had a higher body weight and small tumor size (Table 1), indicating the potential of licorice in improving quality of life of tumor hosts as well as suppressing tumor growth.. This was also evident by the behaviour of treated mice, such as, grooming, feeding and fur appearance. (Data not shown). This is contrary to common effects of conventional therapies that are associated with poor prognosis and response to drugs [32-34]. Tumor suppression has been fronted as a good indicator in cancer prognosis. In relation to tumor suppression, CAMs with anticancer compounds inhibit tumor progression $[35,36]$. This can be attributed to either direct cytotoxicity to cancer cells or it could be through a cascade of events, including immunomodulation. In this study, fraction B treated group showed a significant tumor suppression rate compared to saline group (Table 1). In addition, relative spleen and thymus index are important indices for nonspecific immunity. Potent immunomodulatory compounds increase the spleen and thymus weight [36-39]. This increase is attributed to stimulation of immune cells in the spleen and thymus. The spleen is actively involved in filtration

Table 3 Effect of Licorice polysaccharides on T lymphocyte population

\begin{tabular}{|c|c|c|c|c|c|c|}
\hline TT Cells, \% & Normal & Saline & Cytoxin & FractionA & Fraction B & Fraction C \\
\hline $\mathrm{CD}^{+}$ & $79.82 \pm 1.1^{*}$ & $72.76 \pm 2.3^{\#}$ & $75.74 \pm 4.7^{\#}$ & $77.61 \pm 0.3^{*}$ & $78.70 \pm 3.4^{*}$ & $71.20 \pm .9^{\#}$ \\
\hline $\mathrm{CD}^{+}$ & $18.55 \pm 0.9^{*}$ & $25.98 \pm 2.4^{\#}$ & $19.62 \pm 4.1^{*}$ & $21.25 \pm 0.3^{*}$ & $20.44 \pm 3.2^{*}$ & $27.05 \pm .6^{\#}$ \\
\hline
\end{tabular}

Data are expressed as mean \pm SD. ${ }^{*} p<0.05$, versus Saline group; ${ }^{\#} p<0.05$ versus Normal group, $n=10$ 

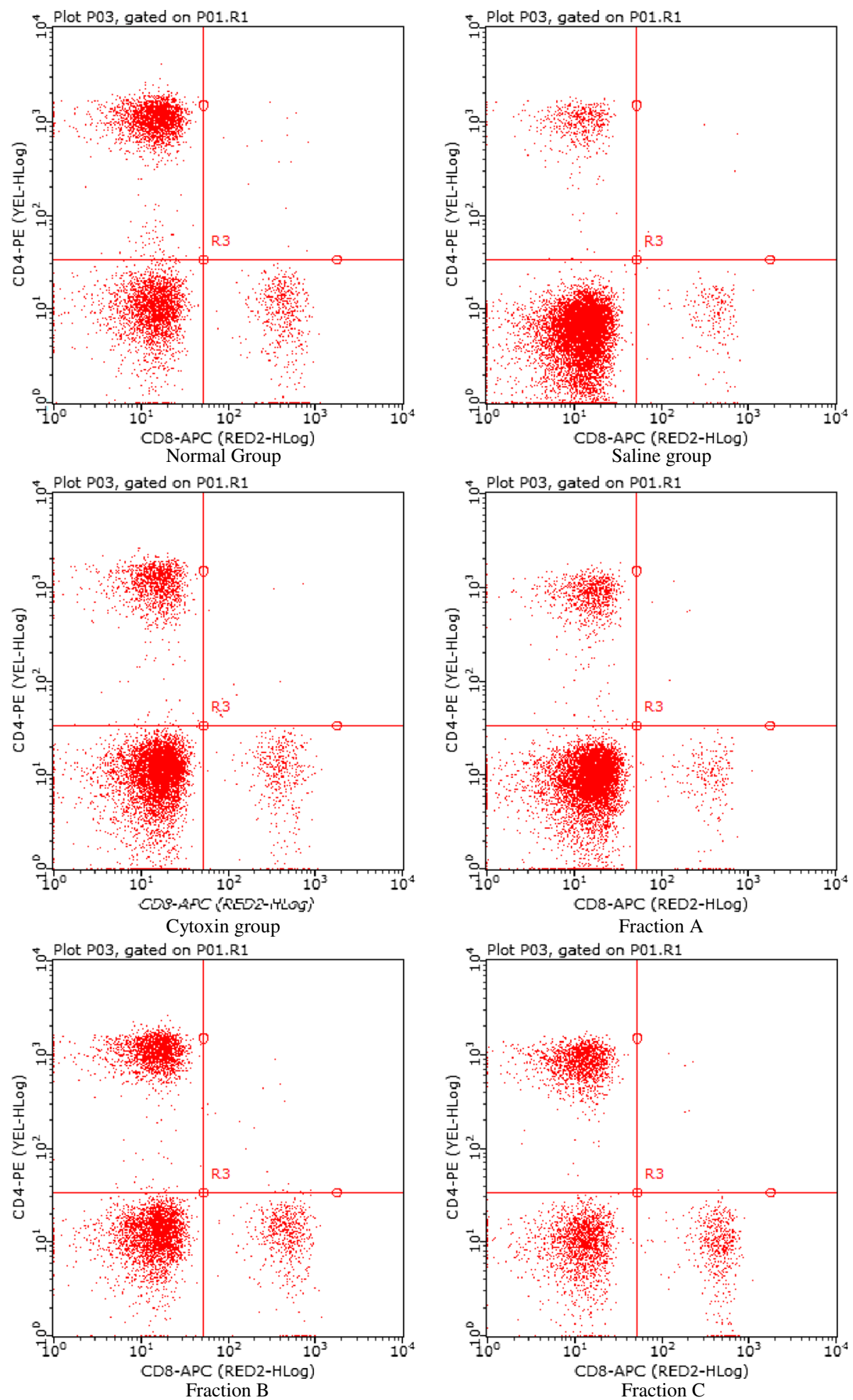

Fig. 1 Dot plots for representative treatment groups of Normal, Saline, Fraction A, Fraction B and Fraction C after treatment period. To determine immune activity of licorice polysaccharides on proliferation T lymphocytes through stimulation, flow cytometry assay was carried out. Whole blood was collected by retro-orbital puncture and stained with respective markers, CD3, CD4 and CD8. Normal group had a high percentage of CD4 and low CD8; Cytoxin group, high CD4 and low CD8; Fraction A, high CD4 and low CD8; Fraction B, high CD4 and low CD8 and Fraction C, low CD4 and high CD8 cells compared to Saline treated group 


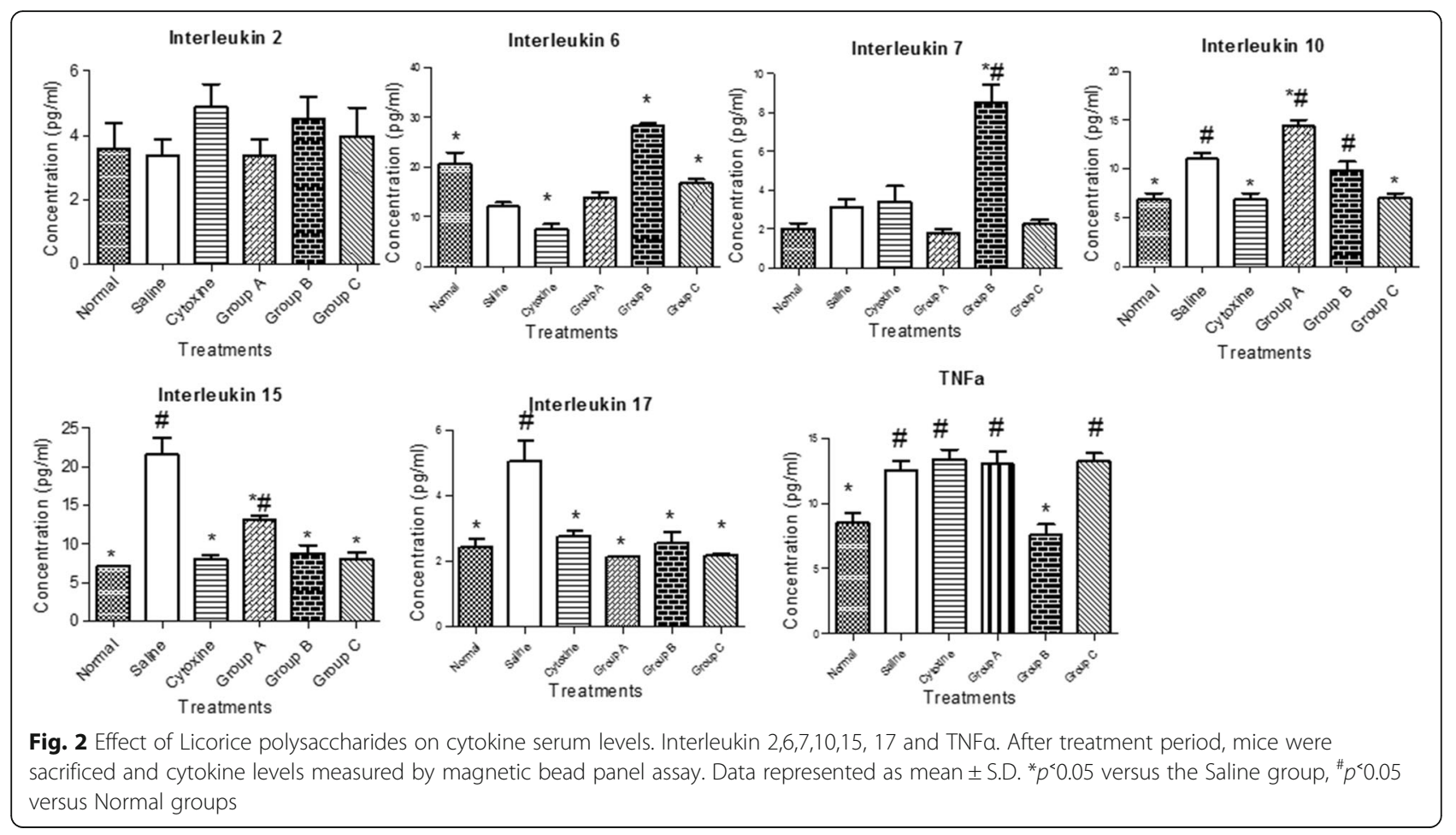

of blood and stores monocytes. Studies indicate that licorice enhances the immune status of mice by increasing blood leukocyte count and weight of the spleen [13]. In our studies, spleen and thymus weight and index were higher in fraction B treated group compared to saline group (Table 2). This phenomenon can be attributed to the immune modulating and anticancer potential of licorice polysaccharides, which is also true with other CAMs [40-42]. The spleen and thymus represent major secondary and primary lymphoid organs involved in eliciting an immune response and maturation of immune cells, and are also important in humoral and cellular immunity. The change in spleen and thymus weight and index can also be attributed to signalling events of IL-7 which is critical in stimulation, maturation and homeostasis of lymphocytes. In our previous studies, we demonstrated the ability of licorice polysaccharides to augment IL-7 expression, secretion and stimulate proliferation of $\mathrm{T}$ lymphocytes, thereby exhibiting immunomodulatory activity on immune organs and cells [10].

It is well documented that one of the probable mechanism of immune stimulation activity of drugs is through activation of immune cells such as CD4 and CD8 $\mathrm{T}$ cells. The increase in these immune cells is thought to be a good indicator of better prognosis and an active immune response to tumors and infections [43]. In the present study, licorice polysaccharide of low molecular weight (Fraction B) stimulated proliferation of CD4 and CD8 T cells compared to saline group (Table 3) with a significant increase and decrease respectively, thus, suggesting that, stimulation and activation of lymphocytes is a probable mechanism of immunomodulation by licorice polysaccharides. This findings concur with other findings that have reported the importance of CD4 T cells in mounting an effective anticancer immunity and reduced $\mathrm{CD} 4 \mathrm{~T}$ cells enhances tumor progression [44, 45]. Moreso, CD4 T cells are known to promote antitumor activity of CD8 T cells, and the antitumor activity is augmented in presence of both CD4 and CD8 T cells [46]. The increased number of CD4 T cells in fraction B treated group, is a likely reason for the observed higher tumor suppression rate. The population of CD4 and CD8 T cells observed again can be associated with stimulation by IL-7 [10, 47].

Furthermore, studies on various anticancer CAMs have demonstrated that promotion of anti-inflammatory cytokines and/or inhibition of pro-inflammatory cytokines is one of their mechanism of action [42, 48]. For instance, IL-7 is significant in cancer immunotherapy, by promoting stimulation, activation, survival and homeostasis of immune effector cells. Due to the potential of IL-7 and other cytokines, some have been approved and are currently used clinically for immunotherapy $[47,49,50]$. In our study, there were differential immunological activities of licorice polysaccharides in CT 26 tumor bearing BALB/C mice. Fraction $B$ upregulated the production of various cytokines such as IL-2, IL-6 and IL-7 in comparison to saline group (Fig. 2). In comparison to normal group, production of IL-10, IL-15 and IL-17 was high but lower than the saline group, while the production of pro-inflammatory 
cytokine, TNF $\alpha$, was lower in fraction B. These cytokines, interleukin-2, IL-6, IL-15 and IL-7 are involved in activating $\mathrm{T}$ lymphocyte growth, survival, maturation and homeostasis. They are therefore, critical cytokines in ensuring effective antitumor immunity [10, 44, 45, 51]. The increased production following treatment with fraction $\mathrm{B}$, exhibits the antitumor and immune modulating activity of these compounds. In fact, lack of IL-7 is associated with severe immunodeficiency and presence of IL-7 in blood cancer patients is an indication of better prognosis which is associated with activating immune effector cells in tumor hosts [51-53]. On the other hand, IL-10, IL-17 and TNF $\alpha$, though some studies indicate that they activate cell growth, they have been associated with blocking apoptosis, inhibiting antigen presenting cells, inhibiting cytokine production, favour tumor growth and promote inflammation. TNF $\alpha$, which is a pro-inflammatory cytokine, also promotes tumor development, neurovascularization and metastasis of tumors $[54,55]$. Low levels of TNF- $\alpha$ in this study, is a good indicator of the antitumor potential of licorice polysaccharides which inhibited its production and circulation, thereby affecting and suppressing tumor growth.

\section{Conclusion}

Current research is geared towards immunotherapy which pose mild side effects. CAMs are also gaining popularity due to their holistic approach involving modulating the immune system and as biological response modifiers (BRMs) in cancer immunotherapy. Licorice polysaccharides of low molecular weight as CAM, have the potential of immunomodulatory and anticancer compounds for cancer treatment. They are non-cytotoxic, suppress tumor growth, increase immune organ weight and index, activate immune cells and stimulate secretion of ant-inflammatory cytokines, especially IL-7, and inhibit secretion of pro-inflammatory cytokines, TNF- $\alpha$. These polysaccharides therefore have a potential of use in cancer immunotherapy.

\section{Abbreviations}

ANOVA: Analyses of variance; CAM: Complementary and Alternative Medicine; CD: Cluster of Differentiation; CT 26: Colon tumor cell line 26; IL: Interleukin; S.E.M: Standard Error of Mean; TCM: Traditional Chinese medicine; TNFa: Tumor Necrotic Factor alpha; TUTCM: Tianjin University of Traditional Chinese medicine

\section{Acknowledgements}

This research was supported by the Chinese Scholarship Council (CSC), through the International Education College, Tianjin University of Traditional Chinese Medicine (TUTCM) PR. China. We gratefully appreciate Xinbo Song for supporting the project. I wish to acknowledge my employer, Egerton University, Njoro, Kenya, for granting me study leave to do research.

\section{Funding}

Authors declare that they have received no funding for this study.

\section{Availability of data and materials}

All data are presented in the manuscript. Datasets used and/or analysed in this study are available from the corresponding author on reasonable request.

\section{Authors' contributions}

BYH designed and supervised the study. PAA designed the study, did experimental work and analyses. PGM, YZ collaborated in carrying out experimental work and analyses. PAA drafted the manuscript. All authors have approved submission of the manuscript.

\section{Ethics approval}

Animal experiments were performed in compliance with the guide for the care and use of laboratory animals, published by the National Institutes of Health (USA). The study was approved by Tianjin University of Traditional Chinese Medicine Animal Care and Use Committee.

\section{Consent for publication}

Not applicable

\section{Competing interests}

The authors declare that they have no competing interests.

\section{Publisher's Note}

Springer Nature remains neutral with regard to jurisdictional claims in published maps and institutional affiliations.

\section{Author details}

${ }^{1}$ International College of Chinese Materia Medica, Tianjin University of Traditional Chinese Medicine, 88 Yuquan Road, 312 Anshan Western Road, Nankai District, Tianjin 300193, People's Republic of China. ${ }^{2}$ Department of Biological Sciences, Faculty of Science, Egerton University, PO BOX 536-20115, Egerton, Kenya. ${ }^{3}$ Center for Traditional Medicine and Drug Research, Kenya Medical Research Institute, P.O. Box 54840-00200, Nairobi, Kenya.

Received: 10 February 2017 Accepted: 28 November 2017

Published online: 15 December 2017

\section{References}

1. Ferlay J, Shin HR, Bray F, Forman D, Mathers C, Parkin DM. Estimates of worldwide burden of cancer in 2008: GLOBOCAN 2008. Int J cancer. 2010; 127(12):2893-917.

2. Torre LA, Bray F, Siegel RL, Ferlay J, Lortet-Tieulent J, Jemal A. Global cancer statistics, 2012. CA Cancer J Clin. 2015;65(2):87-108.

3. Collaboration GBoDC. The global burden of cancer 2013. JAMA Oncol. 2015;1(4):505.

4. Chen Daniel S, Mellman I. Oncology meets immunology: the cancerimmunity cycle. Immunity. 2013;39(1):1-10.

5. Grivennikov SI, Greten FR, Karin M. Immunity, inflammation, and cancer. Cell. 2010;140(6):883-99.

6. Vanneman M, Dranoff G. Combining immunotherapy and targeted therapies in cancer treatment. Nat Rev Cancer. 2012;12(4):237-51.

7. de Visser KE, Eichten A, Coussens LM. Paradoxical roles of the immune system during cancer development. Nat Rev Cancer. 2006;6(1):24-37.

8. Zhang Q, Ye M. Chemical analysis of the Chinese herbal medicine Gan-Cao (licorice). J Chromatogr A. 2009;1216(11):1954-69.

9. Kang HR, Seo JY, Kim SS, Kim BR, Zakharova A, Woo JE, Kim MH, Han MK, Kim J-S: Anti-inflammatory activities of licorice-derived prenylflavonoids (830.30). FASEB J. 2014;28(Suppl 1):830-30.

10. Ayeka PA, Bian Y, Mwitari PG, Chu X, Zhang Y, Uzayisenga R, Otachi EO. Immunomodulatory and anticancer potential of Gan cao (Glycyrrhiza Uralensis Fisch.) polysaccharides by CT-26 colon carcinoma cell growth inhibition and cytokine IL-7 upregulation in vitro. BMC Complement Altern Med. 2016;16(1):1-8.

11. Jo EH, Kim SH, Ra JC, Kim SR, Cho SD, Jung JW, Yang SR, Park JS, Hwang JW, Aruoma Ol, et al. Chemopreventive properties of the ethanol extract of chinese licorice (Glycyrrhiza Uralensis) root: induction of apoptosis and G1 cell cycle arrest in MCF-7 human breast cancer cells. Cancer Lett. 2005; 230(2):239-47.

12. Bode AM, Dong Z. Chemopreventive effects of licorice and its components. Curr Pharmacol Rep. 2015;1(1):60-71.

13. Mitra Mazumder P, Pattnayak S, Parvani H, Sasmal D, Rathinavelusamy P. Evaluation of immunomodulatory activity of $<\mathrm{i}>$ Glycyrhiza glabra $</ \mathrm{i}>\mathrm{L}$ roots in combination with zing. Asian Pac J Trop Biomed. 2012;2(1):S15-20. 
14. Sasaki H, Suzuki N, Alshwaimi E, Xu Y, Battaglino R, Morse L, Stashenko P. 18beta-glycyrrhetinic acid inhibits periodontitis via glucocorticoidindependent nuclear factor-kappaB inactivation in interleukin-10-deficient mice. J Periodontal Res. 2010;45(6):757-63.

15. Hendricks JM, Hoffman C, Pascual DW, Hardy ME: 18ß-glycyrrhetinic acid delivered orally induces isolated lymphoid follicle maturation at the intestinal mucosa and attenuates rotavirus shedding. 2012.

16. Ma J, Peng W, Liang D, Fu N, Pang D, Xu A. The extract of glycyrrhiza uralensis fisch induces apoptosis in MGC-803 cells and its molecular mechanisms. Biomed Res. 2000;21(3):129-37.

17. Zhou Y, Ho WS: Combination of liquiritin, isoliquiritin and isoliquirigenin induce apoptotic cell death through upregulating p53 and p21 in the A549 non-small cell lung cancer cells. Oncol Rep. 2014;31(1):298-304.

18. Park SY, Kwon SJ, Lim SS, Kim J-K, Lee KW, Park JHY. Licoricidin, an active compound in the hexane/ethanol extract of Glycyrrhiza Uralensis, inhibits lung metastasis of 4T1 Murine mammary carcinoma cells. Int J Mol Sci. 2016:17(6):934.

19. Seon MR, Park SY, Kwon SJ, Lim SS, Choi HJ, Park H, Kim J-S, Lee CH, Kim J, Park JHY. Hexane/ethanol extract of Glycyrrhiza uralensis and its active compound isoangustone A induce G1 cycle arrest in DU145 human prostate and 4T1 murine mammary cancer cells. J Nutr Biochem. 2012;23(1): 85-92.

20. Lee CK, Park KK, Lim SS, Park JHY, Chung WY. Effects of the licorice extract against tumor growth and cisplatin-induced toxicity in a mouse xenograft model of colon cancer. Biol Pharm Bull. 2007;30(11):2191-5.

21. Huo HZ, Wang B, Liang YK, Bao YY, Gu Y. Hepatoprotective and antioxidant effects of licorice extract against CCl4-induced oxidative damage in rats. Int J Mol Sci. 2011;12(10):6529-43.

22. Yu J-Y, Ha JY, Kim K-M, Jung Y-S, Jung J-C, Oh S. Anti-inflammatory activities of licorice extract and its active compounds, glycyrrhizic acid, liquiritin and liquiritigenin, in BV2 cells and mice liver. Molecules. 2015;20(7):13041-54

23. Hildebrandt IJ, Su H, Weber WA. Anesthesia and other considerations for in vivo imaging of small animals. ILAR J. 2008;49(1):17-26.

24. Hall RD, Gray JE, Chiappori AA. Beyond the standard of care: a review of novel immunotherapy trials for the treatment of lung cancer. Cancer Control. 2013;20(1):22-31.

25. DeSantis $C E$, Lin CC, Mariotto AB, Siegel RL, Stein KD, Kramer JL, Alteri R, Robbins AS, Jemal A. Cancer treatment and survivorship statistics, 2014. CA Cancer J Clin. 2014;64(4):252-71.

26. Kir S, White JP, Kleiner S, Kazak L, Cohen P, Baracos VE, Spiegelman BM. Tumour-derived PTH-related protein triggers adipose tissue browning and cancer cachexia. Nature. 2014:513(7516):100-4

27. Maria MA, Cristina Z, Laura P, Camillo F, Elena S, PierGiacomo C-P. Cutaneous melanoma in patients in treatment with biological therapy: review of the literature and case report. Dermatol Online J. 2011;17(8):12-12.

28. Johnson M. Chemotherapy treatment decision making by professionals and older patients with cancer: a narrative review of the literature. Eur J Cancer Care. 2012;21(1):3-9

29. Lezoche G, Paganini A, Campagnacci R, Ghiselli R, Pelloni M, Rombini A Guerrieri M. Treatment of rectal cancer by transanal endoscopic microsurgery: review of the literature. Minerva Chir. 2013;68(1):1-9.

30. Munshi A, Agarwal JP, Pandey KC. Cancer patients with cardiac pacemakers needing radiation treatment: a systematic review. J Cancer Res Ther. 2013;9(2):193.

31. Siegel R, Ma J, Zou Z, Jemal A: Cancer statistics, 2014. CA Cancer J Clin. 2014; 64(1):9-29.

32. Kanti V, Nuwayhid R, Lindner J, Hillmann K, Stroux A, Bangemann N, KleineTebbe A, Blume-Peytavi U, Garcia Bartels N. Analysis of quantitative changes in hair growth during treatment with chemotherapy or tamoxifen in patients with breast cancer: a cohort study. Br J Dermatol. 2014;170(3):643-50.

33. Trüeb R. Chemotherapy-induced hair loss. Skin Ther Lett. 2010;15(7):5-7.

34. Paus R, Haslam IS, Sharov AA, Botchkarev VA. Pathobiology of chemotherapy-induced hair loss. Lancet Oncol. 2013;14(2):e50-9.

35. Li X-L, Zhou A-G. Evaluation of the immunity activity of glycyrrhizin in AR mice. Molecules. 2012;17(1):716-27.

36. Zhang S, Nie S, Huang D, Li W, Xie M. Immunomodulatory effect of Ganoderma atrum polysaccharide on CT26 tumor-bearing mice. Food Chem. 2013;136(3):1213-9.

37. Lee KY, Jeon YJ. Macrophage activation by polysaccharide isolated from Astragalus Membranaceus. Int Immunopharmacol. 2005;5(7-8):1225-33.
38. Cheng A, Wan F, Wang J, Jin Z, Xu X. Macrophage immunomodulatory activity of polysaccharides isolated from Glycyrrhiza Uralensis fish. Int Immunopharmacol. 2008;8(1):43-50

39. Li J, Huston G, Swain SL. IL-7 promotes the transition of CD4 effectors to persistent memory cells. J Exp Med. 2003;198(12):1807-15.

40. Wang N, Yang J, Lu J, Qiao Q, Wu T, Du X, Bao G, He X. A polysaccharide from salvia miltiorrhiza Bunge improves immune function in gastric cancer rats. Carbohydr Polym. 2014;111:47-55.

41. Wang X, Song Z-J, He X, Zhang R-Q, Zhang C-F, Li F, Wang C-Z, Yuan C-S. Antitumor and immunomodulatory activity of genkwanin on colorectal cancer in the APCMin/+ mice. Int Immunopharmacol. 2015:29(2):701-7.

42. Yang B, Xiao B, Sun T. Antitumor and immunomodulatory activity of Astragalus Membranaceus polysaccharides in H22 tumor-bearing mice. Int J Biol Macromol. 2013;62:287-90

43. Brush J, Mendenhall E, Guggenheim A, Chan T, Connelly E, Soumyanath A Buresh R, Barrett R, Zwickey H. The effect of Echinacea Purpurea, Astragalus Membranaceus and Glycyrrhiza Glabra on CD69 expression and immune cell activation in humans. Phytother Res. 2006;20(8):687-95.

44. Marzo AL, Kinnear BF, Lake RA, Frelinger JJ, Collins EJ, Robinson BW, Scott B. Tumor-specific CD4+ T cells have a major "post-licensing" role in CTL mediated anti-tumor immunity. J Immunol. 2000;165(11):6047-55.

45. Assudani DP, Horton RB, Mathieu MG, McArdle SE, Rees RC: The role of CD4 $+T$ cell help in cancer immunity and the formulation of novel cancer vaccines. Cancer Immunol Immunother. 2007;56(1):70-80.

46. Gyobu H, Tsuji T, Suzuki Y, Ohkuri T, Chamoto K, Kuroki M, Miyoshi H, Kawarada Y, Katoh H, Takeshima T. Generation and targeting of human tumor-specific Tc1 and Th1 cells transduced with a lentivirus containing a chimeric immunoglobulin T-cell receptor. Cancer Res. 2004;64(4):1490-5.

47. Gao J, Zhao L, Wan YY, Zhu B. Mechanism of action of IL-7 and its potential applications and limitations in cancer immunotherapy. Int J Mol Sci. 2015; 16(5):10267-80.

48. Jayaprakasam B, Yang N, Wen M-C, Wang R, Goldfarb J, Sampson H, Li X-M: Constituents of the anti-asthma herbal formula ASHMI ${ }^{\mathrm{TM}}$ synergistically inhibit IL-4 and IL-5 secretion by murine Th2 memory cells, and eotaxin by human lung fibroblasts in vitro. J Intern Med. 2013;11(3):195-205.

49. Fritzell $S$, Eberstål $S$, Sandén $E$, Visse $E$, Darabi A, Siesjö P. IFNy in combination with IL-7 enhances immunotherapy in two rat glioma models. J Neuroimmunol. 2013;258(1):91-5.

50. Zhao L, Mei Y, Sun Q, Guo L, Wu Y, Yu X, Hu B, Liu X, Liu H. Autologous tumor vaccine modified with recombinant new castle disease virus expressing IL-7 promotes antitumor immune response. J Immunol. 2014; 193(2):735-45

51. Lee S, Margolin K: Cytokines in Cancer Immunotherapy. Cancers. 2011;3(4): 3856-93.

52. von Freeden-Jeffry U, Vieira P, Lucian LA, McNeil T, Burdach SE, Murray R. Lymphopenia in interleukin (IL)-7 gene-deleted mice identifies IL-7 as a nonredundant cytokine. J Exp Med. 1995;181(4):1519-26.

53. Pellegrini M, Calzascia T, Elford AR, Shahinian A, Lin AE, Dissanayake D, Dhanji S, Nguyen LT, Gronski MA, Morre M: Adjuvant IL-7 antagonizes multiple cellular and molecular inhibitory networks to enhance immunotherapies. Nat Med. 2009;15(5):528-36.

54. Mantovani A, Allavena P, Sica A, Balkwill F. Cancer-related inflammation. Nature. 2008:454(7203):436-44

55. Balkwill F. Tumour necrosis factor and cancer. Nat Rev Cancer. 2009; 9(5):361-71.

\section{Submit your next manuscript to BioMed Central and we will help you at every step:}

- We accept pre-submission inquiries

- Our selector tool helps you to find the most relevant journal

- We provide round the clock customer support

- Convenient online submission

- Thorough peer review

- Inclusion in PubMed and all major indexing services

- Maximum visibility for your research

Submit your manuscript at www.biomedcentral.com/submit 